\title{
LA FAMILIA ASTERACEAE EN EL PARQUE NACIONAL LOS MÁRMOLES, HIDALGO, MÉXICO
}

\author{
Carlos Alberto García-SÁnchez ${ }^{1}$, Arturo Sánchez-GonzÁlez ${ }^{1,3}$ \\ Y JoSÉ LUIS VILLASEÑOR ${ }^{2}$
}

\begin{abstract}
${ }^{1}$ Universidad Autónoma del Estado de Hidalgo, Centro de Investigaciones
Biológicas, (UAEH), Ciudad Universitaria; Carretera Pachuca-Tulancingo km. 4.5, 42184 Mineral de la Reforma, Hidalgo, México.

${ }^{2}$ Universidad Nacional Autónoma de México, Instituto de Biología, Departamento de Botánica, Apdo. postal 70-233, 04510 México, D.F., México.

${ }^{3}$ Autor para la correspondencia: arturosg@uaeh.edu.mx
\end{abstract}

\section{RESUMEN}

Se realizó un inventario de las especies de la familia Asteraceae presentes en el Parque Nacional Los Mármoles (PNM), localizado en la porción noroeste del estado de Hidalgo. En el PNM se reconocen seis tipos de vegetación, predominando los bosques de Pinus-Quercus y de Quercus. Se encontraron 101 especies, distribuidas en 13 tribus y 51 géneros; dos de ellas naturalizadas (exóticas). Los miembros más numerosos son de las tribus Eupatorieae (26), Heliantheae (17), Astereae (11) y Coreopsideae (10). Los géneros mejor representados son Ageratina (11), Stevia (11), Pseudognaphalium (6) y Dahlia (5). Las especies endémicas al territorio de la República constituyen el componente más importante (54), siguiendo en relevancia las distribuidas de México a Centroamérica (16); solamente cinco (incluyendo las dos introducidas) también existen en el Viejo Mundo. El uso de estimadores no paramétricos indicó que el grado de completitud del inventario de la flora sinanterológica conocida hasta la fecha para el Parque es de entre 70 y $84 \%$.

Palabras clave: área natural protegida, Asteraceae, completitud, Hidalgo, inventario florístico, México.

\section{ABSTRACT}

An inventory of the species of Asteraceae occurring in the Los Mármoles National Park (PNM) was carried out; this park is located in the northwestern part of the state of 
Hidalgo. In the PNM, six different vegetation types are found, the Pinus-Quercus and Quercus forests being the dominating ones. A total of 101 species of Asteraceae was recorded, distributed in 13 tribes and 51 genera; two species are alien (exotics) for the flora of Mexico. Members of the tribes Eupatorieae (26 species), Heliantheae (17), Astereae (11), and Coreopsideae (10) predominated. The genera with the highest number of species are Ageratina (11), Stevia (11), Pseudognaphalium (6), and Dahlia (5). Species endemic to Mexico constitute the main component (54), followed by those distributed from Mexico to Central America (16); only five species (including the two exotics) also occur in the Old World. The use of non-parametric estimators indicated that the degree of completeness of the Asteraceae flora known to date for the park ranges between 70 and $84 \%$.

Key words: Asteraceae, completeness, floristic inventory, Hidalgo, Mexico, natural protected area.

\section{INTRODUCCIÓN}

La familia Asteraceae o Compositae, constituye el grupo vegetal más diverso de plantas vasculares sobre el planeta (Bremer, 1994; Smith et al., 2004); su distribución es prácticamente cosmopolita y es una de las familias más comunes en la mayor parte de los hábitats (Rzedowski, 1972; Villaseñor, 1993). Asteraceae representa un grupo natural (Funk et al., 2005, 2009), con un número elevado de especies y amplia variación en cuanto a formas de vida, estructura floral, mecanismos de polinización y dispersión de semillas. Sus miembros son fácilmente reconocidos por sus inflorescencias dispuestas en capítulos, sus flores gamopétalas y por lo general pentámeras, cáliz modificado en un vilano, estambres singenesios y el ovario ínfero bicarpelar que deriva en una cipsela (Villaseñor, 1993; Bremer, 1994).

La riqueza de la familia a nivel mundial se estima entre 1,500 y 1,700 géneros y entre 24,000 y 30,000 especies (Bremer, 1994; Katinas et al., 2007; Funk et al., 2009), con centros de diversificación importantes en la región del Mediterráneo en el Viejo Mundo, la región del Cabo en África, Australia, México y la Cordillera de los Andes en Sudamérica (Funk et al., 2005). En el caso particular de México, Villaseñor (2003) y Villaseñor et al. (2007) mencionan la existencia de 361 géneros y 3,012 especies; es decir la concentración más cuantiosa de este grupo de plantas de todos los países del mundo (Ortiz et al., 1998; Villaseñor et al., 2004).

Con respecto al estado de Hidalgo, Villaseñor (datos no publicados) estima la riqueza de Asteraceae en 156 géneros y 542 especies. A nivel local existen algunos 
estudios florísticos en distintas regiones, municipios y áreas naturales protegidas de la entidad que incluyen información sobre esta familia. Destacan los realizados en el matorral xerófilo, en los bosques de coníferas y de Quercus, de la Sierra de Pachuca (Barrios-Rodríguez y Medina-Cota, 1996); y en los bosques mesófilos de montaña de los municipios de Tlanchinol (Luna-Vega et al., 1994), Tenango de Doria (Alcántara-Ayala y Luna-Vega, 1997), Molango (Mayorga et al., 1998), Eloxochitlán (Alcántara-Ayala y Luna-Vega, 2001) y Lolotla (Ponce-Vargas et al., 2006).

En el estado de Hidalgo existen cuatro áreas naturales protegidas (ANP) de jurisdicción federal: el Parque Nacional Tula $(99.5$ ha), el Parque Nacional El Chico (2,739 ha), el Parque Nacional Los Mármoles (23,150 ha) y la Reserva de la Biosfera Barranca de Metztitlán (96,042.94 ha). Los planes de manejo de estas ANP (excepto para el Parque Nacional Tula), publicados por la Comisión Nacional de Áreas Naturales Protegidas (Anónimo, 2003, 2006, 2007) contienen listas florísticas generales, en donde también se incluyen especies de Asteraceae.

Los inventarios florísticos realizados recientemente en el PNM con pteridofitas (Ramírez-Cruz et al., 2009), encinos (Álvarez et al., 2010) y musgos (DelgadilloMoya et al., 2011) indican que el estudio previo justificativo para la re-categorización del parque (Anónimo, 2007) fue parcial, porque en las tres publicaciones mencionadas se encontró un número más elevado de especies. Aunado a ello, las presiones ejercidas por las actividades humanas están conduciendo a la pérdida acelerada de la flora y vegetación del parque (Anónimo, 2007; Sánchez-González et al., 2010). Por tal razón, los objetivos del presente trabajo fueron: (1) obtener información sobre la composición taxonómica de la familia Asteraceae en el PNM, que sirva en un futuro para establecer programas adecuados de conservación y aprovechamiento de los recursos vegetales en esta área natural protegida; y (2) comparar la riqueza de especies de Asteraceae del PNM con la de otras regiones dentro del estado de Hidalgo, con el fin de reconocer los patrones de concentración de la diversidad de esta familia.

\section{ÁREA DE ESTUDIO}

El Parque Nacional Los Mármoles se encuentra en la porción noroeste del estado de Hidalgo, dentro de la Sierra Madre Oriental. Comprende parte de los territorios de los municipios de Jacala de Ledesma, Nicolás Flores, Pacula y Zimapán. Sus coordenadas extremas son $20^{\circ} 45^{\prime} 39^{\prime \prime}$ y $20^{\circ} 58^{\prime} 22^{\prime \prime}$ de latitud norte y $99^{\circ} 08^{\prime} 57^{\prime \prime}$ y 99 $18^{\prime} 39^{\prime \prime}$ de longitud oeste (Fig. 1). El intervalo altitudinal en el área va de los 1500 a los $2820 \mathrm{~m}$; posee una topografía accidentada, con laderas empinadas y barrancas 
profundas; dos desniveles importantes son la Barranca de San Vicente, con una profundidad de $600 \mathrm{~m}$ y una altitud en el fondo de aproximadamente $1700 \mathrm{~m}$, y el Cerro del Cangandhó que alcanza los 2820 m s.n.m. (Anónimo, 2007; Álvarez et al., 2010).

Las rocas que afloran en Los Mármoles son sedimentarias marinas (constituidas por calizas y lutitas), metamórficas, como las pizarras e ígneas intrusivas (granodiorita-diorita) y extrusivas. Hay tres formaciones principales: "Las Trancas" que pertenece al Jurásico Superior, "El Doctor" del Cretácico Medio y "Agua Nueva" del Cretácico Superior (Anónimo, 1994). Los suelos predominantes son de tipo Feozem, Litosol, Luvisol, Regosol y Rendzinas. El PNM forma parte de la región hidrológica denominada cuenca del bajo Río Pánuco (Anónimo, 2007).

Los principales tipos de vegetación que se encuentran en el PNM, de acuerdo con la nomenclatura de Rzedowski (1978) son: el bosque mixto, con codominancia de especies de Pinus y Quercus, que ocupa alrededor de $39.7 \%$ de la superficie del parque; el bosque de Quercus (cubre 21.1\% de la superficie), donde las especies más

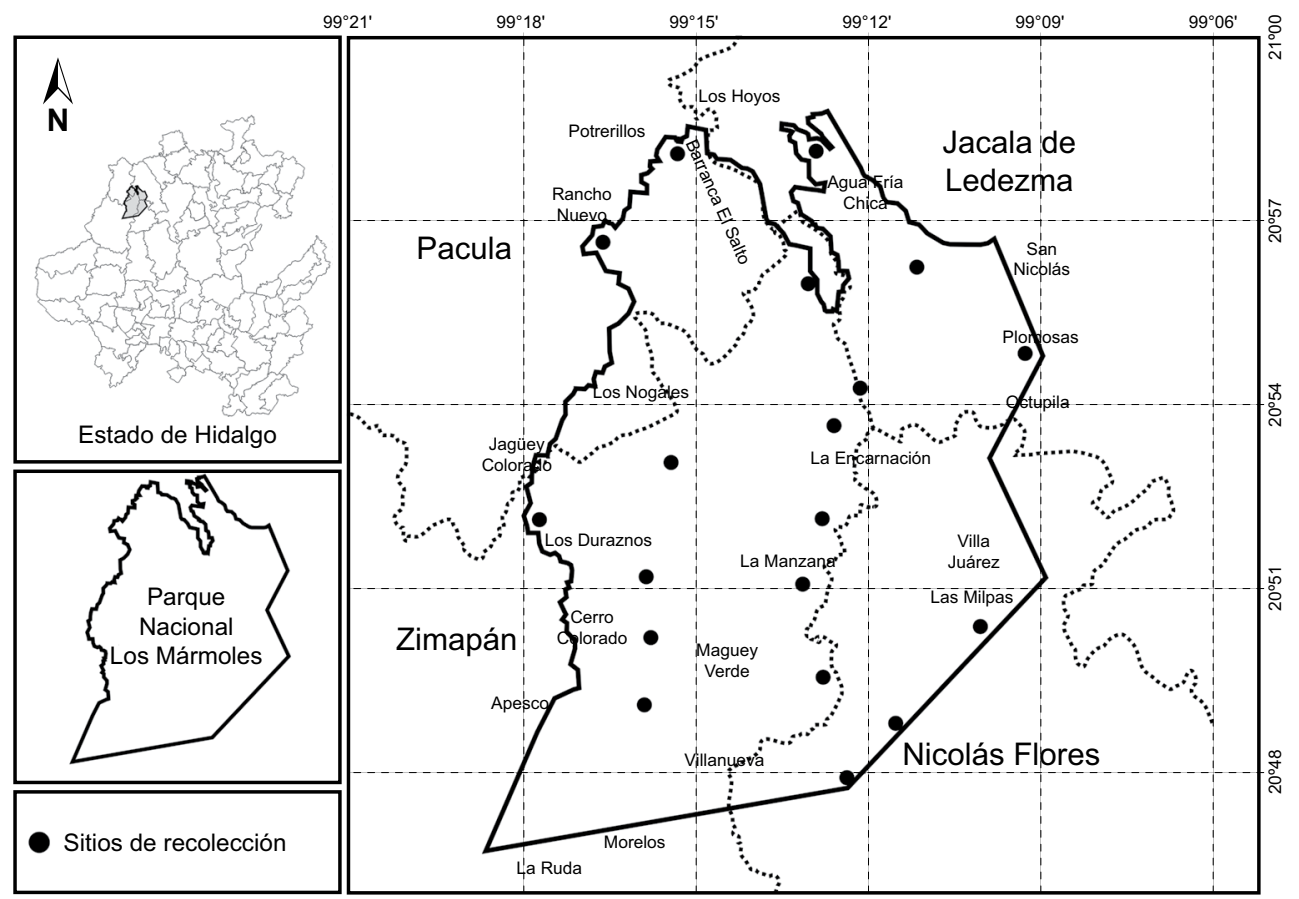

Fig. 1. Localización del Parque Nacional Los Mármoles, estado de Hidalgo, México y de los sitios de recolección. 
comunes son Quercus affinis Scheidw., Q. laeta Liebm., Q. mexicana Humb. \& Bonpl. y Q. rugosa Née (Anónimo, 2007; Álvarez et al., 2010); el bosque de Pinus (6.9\%), que presenta dos tipos de asociaciones importantes, una formada por Pinus cembroides Zucc. y Juniperus deppeana Steud. y otra por P. greggii Engelm. ex Parl., P. teocote Schiede ex Schltdl. \& Cham., P. patula Schiede ex Schltdl. \& Cham. y P. pseudostrobus Lindl.; y el bosque de Juniperus (3.7\% de la superficie), donde dominan $J$. flaccida Schltdl. y $J$. deppeana. La vegetación secundaria de matorral y pastizal, resultado de la actividad humana, ocupa alrededor de $3.6 \%$ del territorio del parque (Anónimo, 2007; Ramírez-Cruz et al., 2009), algunas de las especies características son: Baccharis conferta Kunth, Bouteloua sp., Leucaena sp., Mimosa aculeaticarpa Ortega, Muhlenbergia microsperma (DC.) Kunth y Opuntia spp.

\section{MÉTODOS}

El trabajo de campo consistió en la recolección de ejemplares en 19 sitios (Fig. 1), durante los años de 2007, 2008 y 2009. Para la elección de los mismos se consideró que fueran representativos de las principales asociaciones vegetales y de las diferentes elevaciones de los cuatro municipios que conforman el PNM. Se definieron seis pisos de altitud (1500-1700 m, 1701-1900 m, 1901-2100 m, 2101-2300 $\mathrm{m}, 2301-2600 \mathrm{~m}$ y 2601-2800 m); en los primeros cinco, la búsqueda y recolecta de especímenes se realizó en promedio en cuatro localidades, que incluían 3-4 diferentes tipos de vegetación, de un total de seis, identificados durante los recorridos de campo (Fig. 2). En el piso de mayor altitud (2600-2800 m), sólo se inventarió una localidad de recolecta dentro del parque, que corresponde a la parte alta del Cerro Cangandhó. Es importante mencionar que en todos los puntos estudiados, aun en los de difícil acceso por las condiciones topográficas, se observaron signos de perturbación a causa de las actividades humanas (extracción de madera, presencia de casas-habitación, pastoreo de ganado y obtención de mármol, principalmente). Las muestras fueron depositadas en el Herbario Nacional, con sede en el Instituto de Biología de la Universidad Nacional Autónoma de México (MEXU) y en el herbario del Centro de Investigaciones Biológicas de la Universidad Autónoma del Estado de Hidalgo (HGOM).

La determinación taxonómica se llevó a cabo con la ayuda de floras, monografías, revisiones de la familia, géneros y especies (McVaugh, 1984; Villaseñor, 1987, Rzedowski y Rzedowski, 2001; Villarreal et al., 2001; Kadereit y Jeffrey, 2007, entre otras). Los ejemplares identificados fueron cotejados con material depositado 
en el herbario MEXU. Los tipos de vegetación se definieron fisonómicamente, tomando en cuenta a las especies arbóreas dominantes, de acuerdo con la nomenclatura de Rzedowski (1978).

Adicionalmente, con los datos de incidencia se determinó el grado de completitud del inventario, comparando la riqueza de especies observada contra la esperada, para ello se utilizaron los estimadores no paramétricos bootstrap y jackknife 1 (el cálculo se realizó con 50 aleatorizaciones), los cuales han proporcionado resultados confiables en varias investigaciones (Colwell y Coddington, 1994; GonzálezOreja et al., 2010). Los análisis se realizaron con el programa EstimateS, versión 7.5 (Colwell, 2005).

Con la finalidad de comparar y ponderar la riqueza de especies de Asteraceae presentes en el PNM, con respecto a la de otras áreas naturales protegidas, regiones o municipios del estado de Hidalgo en donde se han realizado inventarios florísticos, se estimó el índice de biodiversidad taxonómica (IB), con la siguiente fórmula: $\mathrm{IB}=$ $\mathrm{S} / \mathrm{Ln} \mathrm{A}$, donde $\mathrm{S}$ es el número de especies registradas y A el tamaño del área (Squeo et al., 1998; Ramírez-Cruz et al., 2009) (ver cuadro 3).

\section{RESULTADOS}

Durante los tres años de trabajo de campo en el PNM, se recolectaron e identificaron un total de 495 ejemplares de compuestas correspondientes a 101 especies, 13 variedades y dos subespecies, ubicadas en 51 géneros y 13 tribus (ver Anexo). De acuerdo con las cifras proporcionadas por Villaseñor (datos no publicados), tales números representan $18.6 \%$ de las especies y $32.7 \%$ de los géneros conocidos para la familia en todo el estado de Hidalgo.

Las tribus que registran el mayor número de géneros son Heliantheae (12), Astereae (7) y Eupatorieae (6); y a nivel de especie Eupatorieae (26), Heliantheae (17), Astereae (11) y Coreopsideae (10) (Cuadro 1). Ageratina y Stevia están representados con 11 cada uno de ellas, Pseudognaphalium con 6 y Dahlia con 5; mientras que los restantes lo están con menos de cuatro (ver Anexo). La proporción especies por género es de dos, cifra menor a la estimada para todo el estado de Hidalgo (3.5).

El número más elevado de taxones (especies, subespecies y variedades) se registró en los bosques de Pinus-Quercus (72) y Quercus (38, Fig. 2), los dos tipos de vegetación con mayor extensión territorial en el parque. En contraste, la menor cantidad se observó en los bosques de Juniperus (con 13), Pinus (13) y Quercus-Juniperus (11), cada uno de los cuales ocupa menos de 7\% de la superficie del PNM (Anónimo, 2007). 
Cuadro 1. Distribución por tribu del número de géneros y especies de Asteraceae en el Parque Nacional Los Mármoles, estado de Hidalgo, México. La clasificación usada es la propuesta por Funk et al. (2009).

\begin{tabular}{lcc}
\hline Tribu & Géneros & Especies \\
\hline Heliantheae & 12 & 17 \\
Astereae & 7 & 11 \\
Eupatorieae & 6 & 26 \\
Senecioneae & 5 & 7 \\
Coreopsideae & 4 & 10 \\
Millerieae & 4 & 4 \\
Lactuceae & 3 & 4 \\
Gnaphalieae & 2 & 8 \\
Neurolaeneae & 2 & 2 \\
Tageteae & 2 & 5 \\
Vernonieae & 2 & 3 \\
Cardueae & 1 & 3 \\
Mutisieae & 1 & 1 \\
\hline
\end{tabular}

Un alto porcentaje de especies en el PNM son consideradas como malezas (31.7\%), lo que indica que la vegetación presenta un importante grado de perturbación; sin embargo, la gran mayoría son nativas del país (Anexo) y únicamente dos, Pseudognaphalium luteo-album (L.) Hilliard \& B.L. Burtt y Sonchus oleraceus L. son introducidas o exóticas (Villaseñor y Espinosa-García, 2004).

La mayoría de las especies $(54,50.5 \%$ y uno de los géneros (Dugesia) son endémicos de México (Cuadro 2). Otro componente importante lo constituyen las especies que se distribuyen de México a Centroamérica (16, 14.9\%). Un porcentaje menor está representado por aquellas que se encuentran en México-Sudamérica $(8,7.5 \%)$, Norteamérica-Sudamérica $(8,7.5 \%)$, México-Norteamérica $(6,5.6 \%)$ y Norteamérica-Centroamérica $(6,5.6 \%)$.

Los estimadores no paramétricos utilizados sugieren niveles satisfactorios de completitud del inventario del PNM; bootstrap indica que la riqueza esperada puede alcanzar hasta 120 especies (desviación estándar $\mathrm{s}=2.57$ ), mientras que con jackknife 1 , puede ser de $143(\mathrm{~s}=11.03)$. Si se comparan ambos valores con relación al observado, la completitud es de $84 \%$ y $70 \%$, respectivamente.

El número de especies de Asteraceae presentes en distintas zonas del estado de Hidalgo difiere marcadamente, independientemente de la extensión territorial 


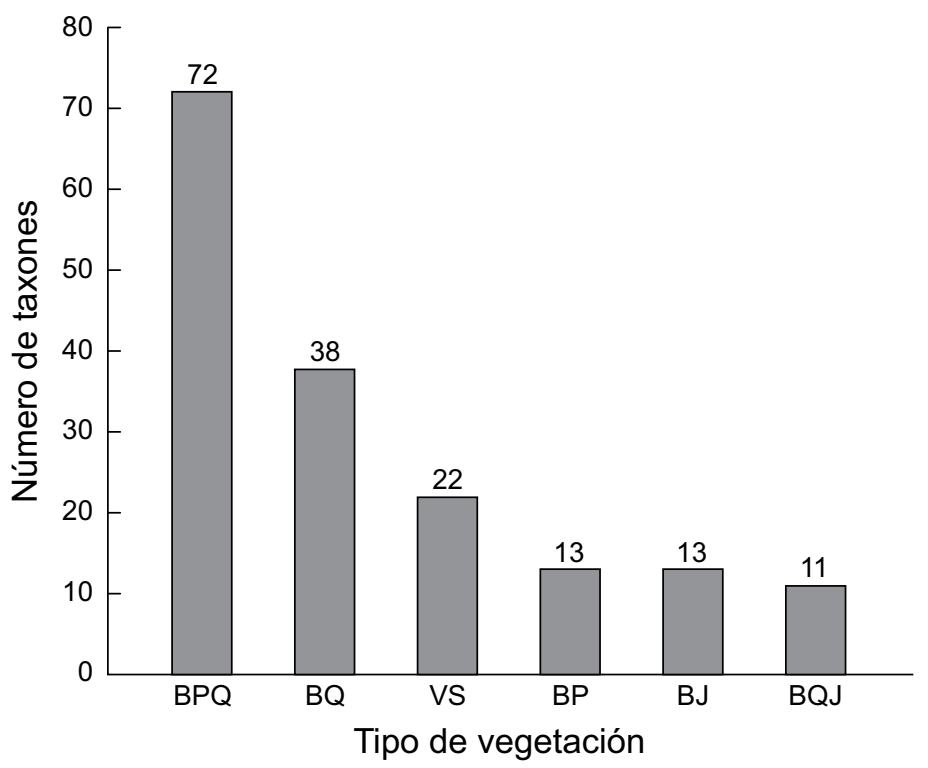

Fig. 2. Riqueza de taxones (especies, subespecies y variedades) de Asteraceae por tipo de vegetación en el Parque Nacional Los Mármoles, estado de Hidalgo. BJ = Bosque de Juniperus, $\mathrm{BP}=$ Bosque de Pinus, $\mathrm{BPQ}=$ Bosque de Pinus-Quercus, $\mathrm{BQ}=$ Bosque de Quercus, BQJ = Bosque de Quercus-Juniperus, VS = Vegetación secundaria.

Cuadro 2. Distribución geográfica de las especies de Asteraceae del Parque Nacional Los Mármoles, estado de Hidalgo.

\begin{tabular}{lc}
\hline Distribución & Número especies \\
\hline Endémicas de México & $54(50.5 \%)$ \\
México a Centroamérica & $16(14.9 \%)$ \\
México a Norteamérica & $6(5.6 \%)$ \\
México a Sudamérica & $8(7.5 \%)$ \\
Norteamérica a Centroamérica & $6(5.6 \%)$ \\
Norteamérica a Sudamérica & $8(7.5 \%)$ \\
Presentes en el Viejo Mundo & $5(4.7 \%)$ \\
Presentes en las islas del Mar Caribe & $4(3.7 \%)$ \\
\hline
\end{tabular}

El número total de especies es mayor de 100, porque algunas se distribuyen en más de una de las regiones geográficas consideradas (ver Anexo). 
de las mismas. La mayor riqueza se concentra en la Sierra de Pachuca, en segundo lugar en el Parque Nacional El Chico y en tercero en el PNM (Cuadro 3).

Cuadro 3. Comparación de las especies de Asteraceae conocidas en distintas Áreas Naturales Protegidas (ANP), regiones y municipios en el estado de Hidalgo. Entre paréntesis se indica el porcentaje de especies con respecto al total registrado para el estado del Hidalgo; IB = índice de biodiversidad taxonómica.

\begin{tabular}{lrrr}
\hline ANP o municipio & \multicolumn{1}{c}{ Especies } & Superficie (ha) & \multicolumn{1}{c}{ IB } \\
\hline 1. Parque Nacional Los Mármoles & $101(18.6 \%)$ & 23150 & 10.05 \\
2. Reserva de la Biosfera Barranca de Metztitlán & $60(11.1 \%)$ & 96042.9 & 5.23 \\
3. Parque Nacional El Chico & $105(19.4 \%)$ & 2739 & 13.26 \\
4. Monte Grande, Lolotla & $36(6.6 \%)$ & 2159 & 4.69 \\
5. Eloxochitlán y Tlahuelompa & $41(7.6 \%)$ & 2000 & 5.39 \\
6. Molocotlán, Molango, Xochicoatlán & $37(6.8 \%)$ & 2467 & 4.74 \\
7. Tenango de Doria & $59(10.9 \%)$ & 2107 & 7.71 \\
8. Tlanchinol & $39(7.2 \%)$ & 3831 & 4.73 \\
9. Sierra de Pachuca & $184(33.9 \%)$ & 60000 & 16.72 \\
10. Estado de Hidalgo & 542 & 2098700 & 37.23 \\
\hline
\end{tabular}

Fuente de información: 1. Anónimo (2007); 2. Anónimo (2003); 3. Anónimo (2006); 4. Ponce-Vargas et al. (2006); 5. Alcántara-Ayala y Luna-Vega (2001); 6. Mayorga et al. (1998); 7. Alcántara-Ayala y Luna-Vega (1997); 8. Luna-Vega et al. (1994); 9. Barrios-Rodríguez y Medina-Cota (1996): 10. Villaseñor (datos no publicados).

\section{DISCUSIÓN}

En el estado de Hidalgo se registran 542 especies de Asteraceae (Villaseñor, datos no publicados); por lo que es la familia más representativa en la entidad, seguida por Poaceae (con 375) y Fabaceae (230) (Sánchez-González et al., 2008). Aunque todavía no se tiene un recuento confiable de la riqueza florística total del PNM, Asteraceae es sin duda también la que posee mayor número de especies (101), cifra que equivale a $18.6 \%$ de toda la diversidad de la familia registrada para el estado de Hidalgo.

Para la Reserva de la Biosfera Barranca de Metztitlán, el área natural protegida con mayor extensión territorial del estado de Hidalgo (96,042.9 ha), en donde predomina el matorral xerófilo y el bosque tropical caducifolio, se mencionan solo 
60 especies de Asteraceae (Anónimo, 2003). En los bosques mesófilos de montaña del estado de Hidalgo, incluidos dentro de la zona templada húmeda de México (sensu Toledo y Ordóñez, 1998), la riqueza de especies de esta familia también es baja (entre 36 y 59). En cambio, en la Sierra de Pachuca (60,000 ha), en el Parque Nacional El Chico (2,739 ha) y en el Parque Nacional Los Mármoles, en donde predominan tipos de vegetación característicos de la zona templada sub-húmeda de México (bosques de Abies, Pinus y Quercus, principalmente), existen 184 (BarriosRodríguez y Medina-Cota, 1996), 105 (Anónimo, 2006) y 101 especies de Asteraceae, respectivamente.

Actualmente, todavía existe la controversia sobre cuál es el grado de contribución del tamaño del área per se y el de otros factores como la productividad, la disponibilidad de energía (clima) y la heterogeneidad ambiental, en la explicación de la riqueza de especies de plantas y animales (Triantis et al., 2008). En el presente estudio, la elevada representación de taxones de Asteraceae en los bosques de Pinus y Quercus, característicos de la zona templada sub-húmeda, sugiere una estrecha correlación con respecto a las condiciones ambientales que predominan en estas comunidades. La propuesta anterior ha sido reiteradamente sugerida, al considerar a la familia como la de mayor diversidad en las regiones templadas sub-húmedas de México, donde puede llegar a representar hasta 15 o $20 \%$ de la flora local o regional (Rzedowski, 1978; Challenger, 1998).

Los estimadores de riqueza representan herramientas estadísticas que permiten tener un panorama objetivo de qué tan completo ha sido el esfuerzo de muestreo o de recolección de ejemplares en el campo. Sin embargo, pueden sub o sobre-estimar el número de especies, por su dependencia con respecto a la frecuencia de las mismas y al tamaño del área de muestreo (Colwell y Coddington, 1994; JiménezValverde y Hortal, 2003; González-Oreja et al., 2010).

Los estimadores utilizados sugieren que el nivel de completitud del inventario se encuentra entre 70 y $84 \%$. De acuerdo con estas cifras, la riqueza esperada de Asteraceae en el PNM podría ser de hasta 143 especies, lo que lo colocaría como una de las regiones del estado de Hidalgo más representativas de la familia. La baja proporción de especies por género en el parque, con respecto a la registrada a nivel estatal, puede considerarse también como un indicio de que el inventario aún es incompleto.

En trabajos previos Sørensen (1969) y Saar et al. (2003) citan a Dahlia cuspidata Saar, P.D. Sørensen \& Hjert., D. mollis P.D. Sørensen y D. moorei Sherff, de varias localidades dentro del PNM, pero en este estudio no se encontraron. Adicionalmente, datos de la Comisión Nacional de Áreas Naturales Protegidas (CONANP) 
(Anónimo, 2007) indican para el PNM la presencia de 62 especies de Asteraceae, de las cuales 34 no se detectaron durante el muestreo en campo. Sin embargo, no se contabilizaron en el inventario por la carencia de ejemplares que respalden su existencia dentro del parque en la actualidad. Un futuro trabajo de campo verificaría su presencia, o la de otras especies todavía no recolectadas, tal y como lo sugieren los modelos predictivos.

Las razones por las que no se encontraron algunas de las especies mencionadas por Sørensen (1969), Saar et al. (2003) o Anónimo (2007), pueden ser: (1) el inventario todavía es incompleto porque no se recorrió todo el PNM, ya que el tamaño del área es muy grande $(23,150 \mathrm{ha})$ y por la inaccesibilidad de algunos sitios con topografía muy accidentada, (2) la recolección de ejemplares no se realizó en todos los meses del año y durante suficiente tiempo (probablemente tres años no sea suficiente), (3) algunas asteráceas que existían en el PNM (como las mencionadas por Sørensen, 1969) han desaparecido a causa de las presiones ejercidas por las actividades humanas, o los fenómenos estocásticos (e.g. incendios forestales, fenómenos naturales) y (4) no se ha revisado críticamente el material incluido en el estudio realizado por la CONANP (Anónimo, 2007), por lo que su presencia en el parque podrá confirmarse cuando los ejemplares botánicos sean depositados en los herbarios.

La mayoría de las plantas identificadas en el presente estudio pertenecen a Astereae, Coreopsideae, Eupatorieae y Heliantheae. Los miembros de estas cuatro tribus prefieren los ambientes templados de las regiones montañosas de México (Faja Volcánica Transmexicana, Sierra Madre del Sur y Sierra Madre Occidental), en donde tienen importantes centros de diversificación (Ryding y Bremer, 1992; Turner y Nesom, 1998). El PNM está situado dentro de la Sierra Madre Oriental, en una zona de convergencia con la Faja Volcánica Transmexicana, por lo que puede considerarse como un área compleja de transición entre provincias florísticas (Delgadillo-Moya et al., 2011), lo que sin duda puede ayudar a explicar la notable riqueza observada de estas y otras tribus dentro de su límites territoriales.

Rzedowski (1978) menciona que Eupatorium, Senecio y Stevia han tenido un importante centro de diversificación en las regiones montañosas de México. En el PNM Ageratina (un segregado de Eupatorium) y Stevia registran el mayor número de especies. Si se considera a Senecio de la manera tradicional, como el único representante de la tribu Senecioneae, también estaría bien representado en el PNM con seis especies; pero ahora sus miembros han sido segregados en varios géneros, por ejemplo Barkleyanthus, Packera, Pittocaulon y Roldana (Kadereit y Jeffrey 2007; Funk et al., 2009). 
Las especies que mostraron una amplia distribución en el PNM son Ageratina ligustrina, Baccharis conferta, Barkleyanthus salicifolius, Bidens odorata y Sonchus oleraceus. La mayoría de ellas toleran bastante bien el disturbio y en varios estudios florísticos realizados en distintos municipios o regiones del estado de Hidalgo, también se ha registrado su presencia (Luna-Vega et al., 1994; Mayorga et al., 1998; Alcántara-Ayala y Luna-Vega, 1997, 2001; Ponce-Vargas et al., 2006). Su amplia distribución podría deberse a una alta plasticidad genética, que les permite adaptarse a diferentes condiciones ecológicas, muchas veces como malezas, como lo sugiere su adaptación a sitios con perturbación (Villaseñor, 1987).

La dominancia de especies exclusivas de México (elemento endémico) y de distribución en regiones vecinas (elemento neártico o mesoamericano) representa un patrón ampliamente discutido de distribución de muchos miembros de la flora de México (Rzedowski, 1991; Villaseñor, 1991). En el caso particular de las asteráceas del PNM, se corrobora el predominio de elementos endémicos a México (ninguna de las especies está restringida al estado de Hidalgo, todas se presentan en dos o más estados de la república mexicana), sobre los que tienen distribución más amplia. Adicionalmente, un elevado porcentaje de las especies sigue el patrón igualmente definido de restringirse a regiones vecinas (suroeste de los Estados Unidos, Guatemala), áreas denominadas por Rzedowski (1991) como Megaméxico.

Reiteradamente se ha mencionado el elevado grado de perturbación de los ambientes del PNM (Ramírez-Cruz et al., 2009; Álvarez et al., 2010; SánchezGonzález et al., 2010; Delgadillo-Moya et al., 2011). El alto porcentaje de especies consideradas malezas $(31.7 \%)$ pudiera indicar efectivamente que la zona está sometida a un fuerte impacto antropogénico (Villaseñor y Espinosa, 1998; Villaseñor y Espinosa-García 2004). A nivel mundial y en México, el proceso de destrucción de los ecosistemas y la consiguiente extinción de las poblaciones de plantas que allí cohabitan está ocurriendo a una tasa acelerada (Sarukhán et al., 2009). Por lo tanto, sigue vigente y urgente la realización de inventarios florísticos, como una de las mejores estrategias para conocer la diversidad existente y de esta manera fundamentar programas de manejo y conservación de los recursos naturales.

\section{AGRADECIMIENTOS}

El trabajo de campo se realizó con fondos económicos del programa de mejoramiento del profesorado (PROMEP), a través del proyecto "Estudio florístico, biogeográfico y sinecológico del Parque Nacional Los Mármoles, Hidalgo, Méxi- 
co" y con el apoyo parcial del convenio FOMIX-Hidalgo, clave 95828 "Diversidad biológica del estado de Hidalgo" (segunda fase). Agradecemos ampliamente los comentarios y sugerencias de dos revisores anónimos, pues sus aportaciones contribuyeron a mejorar sustancialmente el presente artículo.

\section{LITERATURA CITADA}

Alcántara-Ayala, O. e I. Luna-Vega. 1997. Florística y análisis biogeográfico del bosque mesófilo de montaña de Tenango de Doria, Hidalgo, México. Anales Inst. Biol. Univ. Nac. México, Ser. Bot. 68: 57-106.

Alcántara-Ayala, O. e I. Luna-Vega. 2001. Análisis florístico de dos áreas con bosque mesófilo de montaña en el estado de Hidalgo, México: Eloxochitlán y Tlahuelompa. Acta Bot. Mex. 54: 51-87.

Álvarez, Z. E., A. Sánchez-González y S. Valencia. 2010. Los encinos del Parque Nacional Los Mármoles, Hidalgo, México. Madera y Bosques 16(4): 55-66.

Anónimo. 1994. Diagnóstico del Parque Nacional Los Mármoles, estado de Hidalgo. Secretaría de Agricultura y Recursos Hidráulicos. Subsecretaría Forestal y de Fauna Silvestre. Consultores en Ecología y Medio Ambiente. México, D.F., México. 55 pp. Anónimo. 2003. Programa de manejo de la Reserva de la Biosfera Barranca de Metztitlán. Comisión Nacional de Áreas Naturales Protegidas. México, D.F., México. 202 pp.

Anónimo. 2006. Programa de conservación y manejo Parque Nacional El Chico. Comisión Nacional de Âreas Naturales Protegidas. México, D.F., México. 182 pp.

Anónimo. 2007. Estudio previo justificativo para la modificación del decreto por el que se pretende re-categorizar el Parque Nacional Los Mármoles como área de protección de flora y fauna. Comisión Nacional de Áreas Naturales Protegidas. México, D.F., México. 89 pp.

Barrios-Rodríguez, M. A. y J. M. Medina-Cota. 1996. Estudio florístico de la Sierra de Pachuca, estado de Hidalgo. Instituto Politécnico Nacional. México, D.F., México. $140 \mathrm{pp}$.

Bremer, K. 1994. Asteraceae. Cladistics and classification. Timber Press. Portland, USA. 752 pp.

Challenger, A. 1998. Utilización y conservación de los ecosistemas terrestres de México. Pasado, presente y futuro. Comisión Nacional para el Conocimiento y Uso de la Biodiversidad - Instituto de Biología, Universidad Nacional Autónoma de México Agrupación Sierra Madre S.C. México, D.F., México. 847 pp.

Colwell, R. K. 2005. Statistical estimation of species richness and shared species from samples. Versión 7.5.0. University of Connecticut. http://viceroy eeb.uconn.edu/estimates.

Colwell, R. K. y J. A. Coddington. 1994. Estimating terrestrial biodiversity through extrapolation. Philos. T. R. Soc. Lond. Ser. B-Biol. Sci. 345: 101-118.

Delgadillo-Moya, C., M. A. Cárdenas-Soriano, V. M. Gálvez-Aguilar y A. SánchezGonzález. 2011. Musgos del Parque Nacional Los Mármoles, Hidalgo, México. Bol. Soc. Bot. Méx. 89: 19-26. 
Funk, V. A., J. B. Randall, S. C. Keeley, R. Chan, L. Watson, B. Gemeinholzer, E. Schilling, J. L. Panero, B. G. Baldwin, N. García-Jacas, A. Sussana y R. K. Jansen. 2005. Everywhere but Antarctica: Using a supertree to understand the diversity and distribution of the Compositae. Biol. Skr. 55: 343-373.

Funk, V. A., A. Susanna, T. F. Steussy y H. E. Robinson. 2009. Classification of Compositae. In: Funk, V. A., A. Susana, T. F. Stuessy y R. J. Bayer (eds.). Systematics, evolution, and biogeography of Compositae. International Association for Plant Taxonomy (IAPT). Viena, Austria. pp. 171-189.

González-Oreja, J. A., A. A. de la Fuente-Díaz-Ordaz, L. Hernández-Santín, D. BuzoFranco y C. Bonache-Regidor. 2010. Evaluación de estimadores no paramétricos de la riqueza de especies. Un ejemplo con aves en áreas verdes de la ciudad de Puebla, México. Anim. Biod. Conserv. 33: 31-45.

Jiménez-Valverde, A. y J. Hortal. 2003. Las curvas de acumulación de especies y la necesidad de evaluar la calidad de los inventarios biológicos. Rev. Ibér. Arac. 8: 151-161.

Kadereit, J. W. y C. Jeffrey. 2007. Flowering plants, eudicots: Asterales In: Kubitzki, K. (ed.). The families and genera of vascular plants. Vol. VIII. Springer. Berlin, Alemania. 635 pp.

Katinas, L., D. G. Gutiérrez, M. A. Grossi y J. V. Crisci. 2007. Panorama de la familia Asteraceae (Compositae) en la República Argentina. Bol. Soc. Argent. Bot. 42: 113-129.

Luna-Vega I., C. S. Ocegueda y O. Alcántara-Ayala. 1994. Florística y notas biogeográficas del bosque mesófilo de montaña del municipio de Tlanchinol, Hidalgo, México. Anales Inst. Biol. Univ. Nac. México, Ser. Bot. 65: 31-62.

Mayorga, R., I. Luna-Vega y O. Alcántara-Ayala. 1998. Florística del bosque mesófilo de montaña de Molocotlán, Molango-Xochicoatlán, Hidalgo, México. Bol. Soc. Bot. Méx. 63: 101-119.

McVaugh, R. 1984. Compositae. In: Anderson, W. R. (ed.). Flora Novo-Galiciana. Vol. 12. The University of Michigan Press. Ann Arbor, USA. 1157 pp.

Ortiz, B. E., J. L. Villaseñor y O. Téllez. 1998. La familia Asteraceae en el estado de Nayarit (México). Acta Bot. Mex. 44: 25-57.

Ponce-Vargas, A., I. Luna-Vega, O. Alcántara-Ayala y C. A. Ruiz-Jiménez. 2006. Florística del bosque mesófilo de montaña de Monte Grande Lolotla, Hidalgo, México. Rev. Mex. Biodiv. 77: 177-190.

Ramírez-Cruz, S., A. Sánchez-González y D. Tejero-Díez. 2009. La pteridoflora del Parque Nacional Los Mármoles, Hidalgo, México. Bol. Soc. Bot. Méx. 84: 35-44.

Ryding, O. y K. Bremer. 1992. Phylogeny, distribution, and classification of the Coreopsideae (Asteraceae). Syst. Bot. 17: 649-659.

Rzedowski, J. 1972. Contribuciones a la fitogeografía florística e histórica de México III. Algunas tendencias en la distribución geográfica y ecológica de las Compositae mexicanas. Cienc. Mex. 27: 123-132.

Rzedowski, J. 1978. Vegetación de México. Ed. Limusa. México, D.F., México. 432 pp.

Rzedowski, J. 1991. Diversidad y orígenes de la flora fanerogámica de México. Acta Bot. Mex. 14: 3-21.

Rzedowski, J. y colaboradores. 2001. Compositae. In: Rzedowski, G. C. de y J. Rzedowski (eds.). Flora fanerogámica del Valle de México. 2a. ed. Instituto de Ecología, A.C. 
y Comisión Nacional para el Conocimiento y Uso de la Biodiversidad. Pátzcuaro, Michoacán, México. pp. 764-975.

Saar, D. E., P. D Sørensen y J. P. Hjerting. 2003. Dahlia campanulata and D. cuspidata (Asteraceae, Coreopsideae): two new species from Mexico. Acta Bot. Mex. 64: 19-29. Sánchez-González, A., E. Álvarez, M. A. Palacios y A. L. Cuevas. 2008. Datos preliminares sobre la flora vascular del estado de Hidalgo. Herreriana 4: 6-8.

Sánchez-González, A., E. Álvarez y J. D. Tejero-Díez. 2010. Richness and distribution patterns of ferns and lycopods in Los Mármoles National Park, Hidalgo, Mexico. J. Torrey Bot. Soc. 137: 373-379.

Sarukhán, J., P. Koleff, J. Carabias, J. Soberón, R. Dirzo, J. Llorente-Bousquets, G. Halffter, R. González, I. March, A. Mohar, S. Anta y J. de la Maza. 2009. Capital natural de México. Síntesis: conocimiento actual, evaluación y perspectivas de sustentabilidad. Comisión Nacional para el Conocimiento y Uso de la Biodiversidad. México, D.F., México. 100 pp.

Smith, N., S. A. Mori, A. Henderson, D. Wm. Stevenson y S. V. Heald (eds.). 2004. Flowering plants of the Neotropics. Princeton University Press. Princeton, USA. 694 pp.

Sørensen, P. D. 1969. Revision of the genus Dahlia (Compositae, Heliantheae-Coreopsidinae). Rhodora 71: 309-416.

Squeo, F. A., L. A. Cavieres, G. Arancio, J. E. Novoa, O. Matthei, C. Marticorena, R. Rodríguez, M. T. K. Arroyo y M. Muñoz. 1998. Biodiversidad de la flora vascular de la región de Antofagasta, Chile. Rev. Chil. Hist. Nat. 71: 571-591.

Toledo, V. M. y M. J. Ordóñez. 1998. El panorama de la biodiversidad de México: Una revisión de los hábitats terrestres. In: Ramamoorthy, T. P., R. Bye, A. Lot y J. Fa (eds.) Diversidad biológica de México: orígenes y distribución. Instituto de Biología, Universidad Nacional Autónoma de México. México, D.F., México. pp. 739-757.

Triantis, K. A., D. Nogués-Bravo, J. Hortal, A. V. P. Borges, H. Adsersen, J. M. FernándezPalacios, B. M. Araújo y R. J. Whittaker. 2008. Measurements of area and the (island) species area relationship: new directions for an old pattern. Oikos 117: 1555-1559.

Turner, B. L. y G. L. Nesom. 1998. Biogeografía, diversidad y situación de peligro o amenaza de Asteraceae de México. In: Ramamoorthy, T. P., R. Bye, A. Lot y J. Fa. (eds.). Diversidad biológica de México: orígenes y distribución. Instituto de Biología, Universidad Nacional Autónoma de México. México, D.F., México. pp. 545-561.

Villarreal, J. A., R. J. Valdés y J. L. Villaseñor. 2001. Clave genérica para las Asteraceas de Coahuila. Biotam 12(2): 31-48.

Villaseñor, J. L. 1987. Clave genérica para las compuestas de la Cuenca del Rio Balsas. Bol. Soc. Bot. Méx. 47: 65-86.

Villaseñor, J. L. 1991. The genera of Asteraceae endemic to Mexico and adjacent regions. Aliso 12: 685-692.

Villaseñor, J. L. 1993. La familia Asteraceae en México. Rev. Soc. Mex. Hist. Nat. 44: 117124.

Villaseñor, J. L. 2003. Diversidad y distribución de las Magnoliophyta de México, Interciencia 28(3): 160-167.

Villaseñor, J. L. y F. J. Espinosa. 1998. Catálogo de malezas de México. Universidad Nacional Autónoma de México-Fondo de Cultura Económica. México, D.F., México. 449 pp. 
Villaseñor, J. L. y F. J. Espinosa-García. 2004. The alien flowering plants of Mexico. Divers. Distrib. 10: 113-123.

Villaseñor, J. L., E. Ortiz y V. Juárez. 2004. Asteraceas. In: García-Mendoza, A. J., M. J. Ordóñez y M. Briones-Salas (eds.). Biodiversidad de Oaxaca. Instituto de Biología, Universidad Nacional Autónoma de México - Fondo Oaxaqueño para la Conservación de la Naturaleza-World Wildlife Fund. México, D.F., México. pp. 177-192.

Villaseñor, J. L., P. Maeda, J. A. Rosell y E. Ortiz. 2007. Plant families as predictors of plant biodiversity in Mexico. Divers. Distrib. 13: 871-876.

Recibido en julio de 2011.

Reactivado en marzo de 2012.

Aceptado en junio de 2013. 


\section{ANEXO}

Lista de especies de Asteraceae presentes en el Parque Nacional Los Mármoles, estado de Hidalgo. BJ = Bosque de Juniperus, BP = Bosque de Pinus, BPQ = Bosque de Pinus-Quercus, BQ = Bosque de Quercus, BQJ = Bosque de QuercusJuniperus VS = Vegetación secundaria. (*) Especies consideradas como malezas, (**) especies exóticas (introducidas), (1) Endémicas de México, (2) México a Centroamérica, (3) México a Norteamérica, (4) México a Sudamérica, (5) Norteamérica a Sudamérica, (6) Norteamérica-Centroamérica, (7) Presentes en el Viejo Mundo, (8) Presentes en las islas del Mar Caribe, $\mathrm{D}=$ distribución geográfica.

\begin{tabular}{|c|c|c|}
\hline Categoría taxonómica & Tipo de vegetación & $\mathrm{D}$ \\
\hline \multicolumn{3}{|l|}{ TRIBU ASTEREAE } \\
\hline Astranthium purpurascens (B.L. Rob.) Larsen & $\mathrm{BPQ}, \mathrm{BQ}, \mathrm{VS}$ & 1 \\
\hline Baccharis conferta Kunth & $\mathrm{BPQ}$ & 2 \\
\hline Baccharis pteronioides DC. & BJ & 3 \\
\hline Erigeron galeottii (A. Gray ex Hemsl.) Greene & BPQ & 1 \\
\hline Erigeron karvinskianus DC. & $\mathrm{BPQ}, \mathrm{BQ}$ & 4,8 \\
\hline Erigeron longipes DC. * & $\mathrm{BPQ}, \mathrm{BQ}$ & 6 \\
\hline Erigeron pubescens Kunth * & $\mathrm{BPQ}, \mathrm{BQ}, \mathrm{VS}$ & 3 \\
\hline Grindelia inuloides Willd. var. inuloides $*$ & $\mathrm{BP}$ & 1 \\
\hline $\begin{array}{l}\text { Gutierrezia texana (DC.) Torr. \& A. Gray var. glutinosa } \\
\text { (Schauer) M.A. Lane }\end{array}$ & BPQ & 3 \\
\hline Heterotheca inuloides Cass. var. inuloides $*$ & $\mathrm{BJ}$ & 1 \\
\hline $\begin{array}{l}\text { Symphyotrichum expansum (Poepp. ex Spreng.) G.L. } \\
\text { Nesom }\end{array}$ & BPQ, VS & 5 \\
\hline \multicolumn{3}{|l|}{ TRIBU CARDUEAE } \\
\hline Cirsium acrolepis (Petr.) G.B. Ownbey & $\mathrm{BPQ}, \mathrm{VS}$ & 1 \\
\hline Cirsium pinetorum Greenm. & $\mathrm{BPQ}, \mathrm{VS}$ & 1 \\
\hline Cirsium rhaphilepis (Hemsl.) Petr. * & BPQ & 1 \\
\hline \multicolumn{3}{|l|}{ TRIBU COREOPSIDEAE } \\
\hline Bidens odorata Cav. var. odorata * & BPQ, BQJ, BQ & 6 \\
\hline Bidens odorata Cav. var. rosea (Sch. Bip.) Melchert & $\mathrm{BQ}, \mathrm{BQJ}$ & 4 \\
\hline Bidens triplinervia Kunth * & BP, BPQ, BQJ & 4 \\
\hline Coreopsis mutica DC. var. mutica * & $\mathrm{BP}, \mathrm{BPQ}, \mathrm{BQ}, \mathrm{VS}$ & 1 \\
\hline Cosmos atrosanguineus (Ortgies) Hemsl. & BPQ & 1 \\
\hline Cosmos bipinnatus Cav. * & $\mathrm{BJ}$ & 5,8 \\
\hline
\end{tabular}


Anexo. Continuación.

\begin{tabular}{lll}
\hline Categoría taxonómica & Tipo de vegetación & D \\
\hline Dahlia coccinea Cav. * & BP, BPQ, BQ, VS & 2 \\
Dahlia merckii Lehm. * & BPQ, BQ, VS & 1 \\
Dahlia rudis P.D. Sørensen & BPQ, BQ, VS & 1 \\
Dahlia scapigeroides Sherff & BPQ, BQ & 1 \\
Dahlia sørensenii H.V. Hansen \& Hjert. * & BPQ, BQ, VS & 1 \\
TRIBU EUPATORIEAE & &
\end{tabular}

\section{TRIBU EUPATORIEAE}

Ageratina brandegeana (B.L. Rob.) R.M. King \& H. Rob. Ageratina calaminthifolia (Kunth) R.M. King \& H. Rob. BQ

Ageratina choricephala (B.L. Rob.) R.M. King \& H. Rob. BQ

Ageratina glabrata (Kunth) R.M. King \& H. Rob.

BJ, BPQ 1

Ageratina hidalgensis (B.L. Rob.) R.M. King \& H. Rob.

Ageratina ligustrina (DC.) R.M. King \& H. Rob.

$\mathrm{VS}$

BPQ, BQ 2

Ageratina pazcuarensis (Kunth) R.M. King \& H. Rob.

BPQ

Ageratina petiolaris (Moç. \& Sessé ex DC.) R.M. King \&

$\mathrm{BJ}, \mathrm{BQ}$

H. Rob.

Ageratina pichinchensis (Kunth) R.M. King \& H. Rob.

Ageratina queretaroana B.L. Turner

BPQ 4

Ageratina rhomboidea (Kunth) R.M. King \& H. Rob.

BPQ

BP

Brickellia veronicifolia (Kunth) A. Gray

BPQ

Fleischmannia pycnocephala (Less.) R.M. King \& H.

BJ, BPQ, BQ

Rob.

Kyrsteniopsis spinaciifolia (DC.) B.L. Turner

BQ, VS

Piqueria trinervia Cav. *

$\mathrm{BJ}, \mathrm{BQ}$

2,8

Stevia berlandieri A. Gray

BPQ

BQ

BP

BPQ

BPQ, BQ

BPQ, BQ

BPQ

BP, BPQ, BQ

Stevia elatior Kunth *

Stevia iltisiana Grashoff

Stevia jorullensis Kunth *

Stevia ovata Willd. var. ovata

Stevia porphyrea McVaugh

Stevia pyrolifolia Schltdl.

Stevia salicifolia Cav. var. salicifolia

BJ

BPQ

Stevia serrata Cav. var. serrata *

BPQ

Stevia tephra B.L. Rob. 
Anexo. Continuación.

\begin{tabular}{|c|c|c|}
\hline Categoría taxonómica & Tipo de vegetación & $\mathrm{D}$ \\
\hline \multicolumn{3}{|l|}{ TRIBU GNAPHALIEAE } \\
\hline Gamochaeta americana (Mill.) Wedd. * & BJ, BPQ & 7 \\
\hline Gamochaeta falcata (Lam.) Cabrera & $\mathrm{BPQ}, \mathrm{BQ}$ & 1 \\
\hline Pseudognaphalium attenuatum (DC.) Anderb. & $\mathrm{BQJ}$ & 6 \\
\hline Pseudognaphalium canescens (DC.) Anderb. & $\mathrm{BPQ}$ & 3 \\
\hline Pseudognaphalium chartaceum (Greenm.) Anderb. & BPQ & 1 \\
\hline $\begin{array}{l}\text { Pseudognaphalium luteo-album (L.) Hilliard \& B.L. } \\
\text { Burtt *,** }\end{array}$ & $\mathrm{BPQ}$ & 7 \\
\hline Pseudognaphalium oxyphyllum (DC.) Kirp. & BPQ & 2 \\
\hline Pseudognaphalium semilanatum (DC.) Anderb. & $\mathrm{BPQ}, \mathrm{BQ}, \mathrm{VS}$ & 1 \\
\hline \multicolumn{3}{|l|}{ TRIBU HELIANTHEAE } \\
\hline Acmella repens (Walter) Rich. & BPQ & 5 \\
\hline Aldama dentata La Llave * & BJ, BPQ, BQ, BQJ & 2 \\
\hline Dugesia mexicana (A. Gray) A. Gray * & BPQ & 1 \\
\hline Jefea lantanifolia (Schauer) Strother & $\mathrm{BQJ}$ & 1 \\
\hline $\begin{array}{l}\text { Montanoa leucantha (Lag.) S.F. Blake subsp. arborescens } \\
\text { (DC.) V.A. Funk }\end{array}$ & VS & 2 \\
\hline Montanoa mollisima Brongn. ex Groenl. & $\mathrm{BQ}, \mathrm{VS}$ & 1 \\
\hline Montanoa tomentosa Cerv. subsp. tomentosa & BPQ & 1 \\
\hline Perymenium berlandieri DC. & $\mathrm{BPQ}$ & 1 \\
\hline Perymenium mendezii DC. var. verbesinoides (DC.) J.J. Fay & VS & 1 \\
\hline Sanvitalia procumbens Lam. * & $\mathrm{BP}, \mathrm{BPQ}, \mathrm{BQ}, \mathrm{VS}$ & 2 \\
\hline $\begin{array}{l}\text { Sclerocarpus uniserialis (Hook.) Benth. \& Hook. f. ex } \\
\text { Hemsl. * }\end{array}$ & BQJ, VS & 6 \\
\hline Verbesina parviflora (Kunth) S.F. Blake & BPQ & 1 \\
\hline $\begin{array}{l}\text { Verbesina robinsonii (Klatt) Fernald ex B.L. Rob \& } \\
\text { Greenm. }\end{array}$ & BQJ, VS & 1 \\
\hline Verbesina tetraptera (Ortega) A. Gray & BPQ & 1 \\
\hline Viguiera adenophylla S.F. Blake & BQ & 1 \\
\hline Zaluzania megacephala Sch. Bip. var. megacephala & BPQ & 1 \\
\hline Zinnia peruviana (L.) L. * & $\mathrm{BQJ}$ & $5,7,8$ \\
\hline \multicolumn{3}{|l|}{ TRIBU LACTUCEAE } \\
\hline Hieracium dysonymum S.F. Blake & BPQ & 1 \\
\hline Hieracium schultzii Fr. & $\mathrm{BPQ}$ & 6 \\
\hline
\end{tabular}


Anexo. Continuación.

\begin{tabular}{|c|c|c|}
\hline Categoría taxonómica & Tipo de vegetación & $\mathrm{D}$ \\
\hline Pinaropappus roseus (Less.) Less. var. roseus * & BPQ, BQ & 3 \\
\hline Sonchus oleraceus L. *,** & $\mathrm{BJ}, \mathrm{BP}, \mathrm{BPQ}$ & 7 \\
\hline \multicolumn{3}{|l|}{ TRIBU MILLERIEAE } \\
\hline Alloispermum scabrum (Lag.) H. Rob. & BP, BQ, BQJ & 2 \\
\hline Galinsoga parviflora Cav. * & BPQ, BQ, BQJ, VS & 7 \\
\hline Jaegeria hirta (Lag.) Less. * & $\mathrm{BPQ}$ & 4 \\
\hline Smallanthus maculatus (Cav.) H. Rob. & $\mathrm{BP}, \mathrm{BPQ}$ & 2 \\
\hline \multicolumn{3}{|l|}{ TRIBU MUTISIEAE } \\
\hline Chaptalia runcinata Kunth & $\mathrm{BPQ}, \mathrm{VS}$ & 4 \\
\hline \multicolumn{3}{|l|}{ TRIBU NEUROLAENEAE } \\
\hline Calea ternifolia Kunth & $\mathrm{BPQ}$ & 2 \\
\hline Tetrachyron websteri (Wussow \& Urbatsch) B.L. Turner & $\mathrm{BP}, \mathrm{BPQ}$ & 1 \\
\hline \multicolumn{3}{|l|}{ TRIBU SENECIONEAE } \\
\hline Barkleyanthus salicifolius (Kunth) H. Rob. \& Brettell * & $\mathrm{BQ}$ & 6 \\
\hline $\begin{array}{l}\text { Packera zimapanica (Hemsl.) C.C. Freeman \& T.M. } \\
\text { Barkley }\end{array}$ & $\mathrm{BQ}, \mathrm{VS}$ & 1 \\
\hline Pittocaulon praecox (Cav.) H. Rob. \& Brettell & $\mathrm{BJ}, \mathrm{BPQ}$ & 1 \\
\hline Psacalium radulifolium (Kunth) H. Rob. \& Brettell & $\mathrm{BPQ}$ & 1 \\
\hline Roldana albonervia (Greenm.) H. Rob. \& Brettell & $\mathrm{BPQ}, \mathrm{BQ}$ & 1 \\
\hline Roldana aschenborniana (Schauer) H. Rob. \& Brettell & $\mathrm{BQ}$ & 2 \\
\hline Roldana barba-johannis (DC.) H. Rob. \& Brettell & BPQ & 2 \\
\hline \multicolumn{3}{|l|}{ TRIBU TAGETEAE } \\
\hline Dyssodia pinnata (Cav.) B.L. Rob var. pinnata & $\mathrm{BP}, \mathrm{BPQ}$ & 1 \\
\hline Tagetes erecta $\mathrm{L} . *$ & $\mathrm{BPQ}$ & 5 \\
\hline Tagetes filifolia Lag. * & $\mathrm{BPQ}$ & 4 \\
\hline Tagetes lucida Cav. * & $\mathrm{BPQ}$ & 2 \\
\hline Tagetes lunulata Ortega * & $\mathrm{BPQ}$ & 1 \\
\hline \multicolumn{3}{|l|}{ TRIBU VERNONIEAE } \\
\hline Vernonanthura liatroides (DC.) H. Rob. & BQ & 1 \\
\hline Vernonia alamanii DC. & $\mathrm{BJ}, \mathrm{BPQ}, \mathrm{BQ}$ & 1 \\
\hline Vernonia greggii A. Gray & $\mathrm{BP}, \mathrm{BQ}$ & 1 \\
\hline
\end{tabular}

